\title{
Patients with HER2-positive Early Breast Cancer Receiving Adjuvant Trastuzumab: Clinicopathological Features, Efficacy, and Factors Affecting Survival
}

\author{
Arife Ulas ${ }^{1 *}$, Tugba Kos ${ }^{2}$, Nilufer $\mathrm{Avci}^{3}$, Erdem Cubukcu ${ }^{4}$, Omer Fatih Olmez \\ Nilufer Bulut ${ }^{4}$, Mustafa Degirmenci ${ }^{4}$
}

\begin{abstract}
Background: The aim of the present study was to evaluate clinicopathological characteristics of our early stage breast cancer patients who are epidermal growth factor receptor 2 (HER2) overexpressed/ amplified (HER2+), the efficacy of trastuzumab treatment and survival results. Materials and Methods: Patients with HER2- positive early stage breast cancer receiving adjuvant trastuzumab were investigated retrospectively. Clinicopathological features of 210 patients and treatment outcome were analysed. To evaluate survival rates, the Kaplan-Meier method was used. Univariate and multivariate analyses were conducted with the Cox regression model. Results: Mean age of the patients was $51.8,71.9 \%$ being postmenopausal. Some $37.6 \%$ of patients were node negative, and $31 \%$ had $\mathrm{T} 1$ tumor size and $52.4 \%$ were positive for estrogen receptor. Of 210 patients, $89.5 \%$ completed planned 52 weeks adjuvant trastuzumab treatment. The median follow up was 27.5 months $(6.0-86.0)$. Relapse free survival (RFS) was 68.0 months (95\% CI: 62.1-74.0) and overall survival (OS) was 74.8 months $(95 \%$ CI: 69.5-80.1). The 3 year OS for all patients was $92.0 \%$ and RFS was $79.6 \%$. During follow up, relapse was detected at the rate of $14.3 \%$. Trastuzumab associated cardiotoxicity was found at the rate of $3.3 \%$. In univariate analyses, larger tumor size and grade III were significantly associated $(\mathbf{p}<0.05)$ with RFS. Multivariate analyses of covariates displaying $\mathbf{p}<\mathbf{0 . 0 5}$ identified grade III as an independent prognostic factor. Conclusions: In the present study, it was established that trastuzumab had a satisfactory safety profile and treatment efficacy as in other clinical studies and that among clinicopathological factors evaluated, only being grade 3 had a significant effect on RFS. The occurrence of relapse with adjuvant trastuzumab makes it necessary to identify molecular predictors, which will define this group better and help explain resistance to anti HER2 based therapies.
\end{abstract}

Keywords: Adjuvant chemotherapy - trastuzumab - HER2 - breast cancer - grade III

Asian Pac J Cancer Prev, 16 (4), 1643-1649

\section{Introduction}

Breast cancer is a heterogenous disease. Gene expresssion studies have identified five distinct molecular subtypes of breast cancer. The use of gene expression profiling is currently limited in many clinical settings, and hence histopathological markers such as estrogen receptor (ER), progesterone receptor (PR), and HER2 are used as surrogates for the moleculer subtypes, which divide breast tumors into distinct phenotypes with distinct outcomes (Perou et al., 2000).

The gene for the human epidermal growth factor receptor2 (HER2) encodes for a transmembrane tyrosine kinase receptor protein. HER2 has both prognostic and predictive importance for invasive breast cancer. In approximately $15-25 \%$ of patients with breast cancer, HER2 gene is amplified and is associated with increased locoregional recurrence and breast cancer mortality (Slamon et al., 2001; Gabos et al., 2010).

Trastuzumab is a humanized monoclonal antibody developed against extracellular domain of HER2. It has been reported that HER2 blockage made with the addition of trastuzumab to standard chemotherapy regimes increases time to progression and overall survival (OS) significantly in patients with metastatic breast cancer (Salamon et al., 2001; Abe et al., 2013; Zhu et al., 2013). Major studies carried out subsequently on adjuvant trastuzumab demonstrated that, in over 13.000 participants, it decreased disease recurrence and improved disease free survival (DFS) and OS (Baselga et al., 2006). Results of the Herceptin Adjuvant (HERA) trial at 2 years of median follow-up demonstrated that 1-year adjuvant trastuzumab treatment significantly improved rates of DFS and OS in comparison to observation group, with a significant benefit

${ }^{1}$ Department of Medical Oncology, ${ }^{1}$ Ankara Ataturk Training and Research Hospital, Ankara, ${ }^{2}$ Faculty of Medicine, Duzce University, Duzce, ${ }^{3}$ Ballkesir Government Hospital, Ballkesir, ${ }^{4}$ Ali Osman Sonmez Oncology Hospital, Bursa, Turkey *For correspondence: drarifeulas@hotmail.com 
still observed at 4-year and 8-year median follow-up (Smith et al., 2007; Gianni et al., 2011; Goldhirsch et al., 2013 ). These findings confirm that adjuvant trastuzumab administered sequentially to chemotherapy is associated with a persistent benefit and remains an appropriate treatment modality in patients with HER2-positive early breast cancer. This effect was not observed only in one study (Spielman et al., 2009). Adjuvant trastuzumab was accepted as standard management in HER2 positive breast cancer patients from 2006.

In phase III studies, it was demonstrated that trastuzumab was well tolerated (Smith et al., 2007; Gianni et al., 2011; Goldhirsch et al., 2013). The main documented adverse event was cardiotoxicity. The risk of cardiotoxicity associated with trastuzumab was addressed in meta analyses (Bria et al., 2008; Dahabreh et al., 2008). In these metanalyses, decreased left ventricle ejection fraction (LVEF) and other cardiac events (including rare cases of symptomatic heart failure) were reported at the rates of $2.2 \%$ to $14 \%$.

In our country, the use of trastuzumab as adjuvant treatment in HER2+ breast cancer patiens was initiated in 2008.The aim of this retrospective study was to evaluate clinicopathological characteristics HER-2 positive early stage breast cancer patients treated according to HERA protocol in routine clinical practice and the efficacy of adjuvant trastuzumab and survival results in these patients.

\section{Materials and Methods}

\section{Patients and Methods}

The present study was carried out in oncology departments of two centers (Bursa Oncology hospital and Balıkesir public hospital, in Turkey) with the approval of ethics committee. HER2-positive early breast cancer patients diagnosed between January 2009 and January 2014 and who underwent resection and received adjuvant trastuzumab were evaluated retrospectively.

210 female patients who receive early stage adjuvant trastuzumab based treatment and who have immunohistochemically (IHC 3+) HER2 or with fluoresence in situ hybridization (FISH ratio $\geq 2$ ) or with silver in situ hybridization (SISH), HER2 gene amplification were enrolled in the present study. HER2 was determined by immunohistochemistry (IHC) and scored from 0 to $3+$. Scoring of $2+$ was assigned when there was weak to moderate complete membrane staining in $>10 \%$ of tumor cells, whereas scoring of $3+$ consisted of uniform intense membrane staining of $>10 \%$ of invasive tumor cells. SISH or FISH analyses were carried out for all HER2 IHC 2+ samples. HER2 was considered amplified if the ratio of HER2 gene chromosome 17 was $\geq 2.0$.

Patients who had metastasis at the time of diagnosis, those who have contralateral breast cancer and cancers other than breast cancer, and the ones who received neoadjuvant treatment were excluded from the study. Demographic characteristics of the patients, their histopathological data, ER and PR status, type of surgery, site of first relapse, and times of relapse, adjuvant chemoterapy and radiotherapy status were recorded.
Staging of the patients was made according to the American Joint Committee on Cancer (AJCC) criteria. Cardiotoxicity developing during treatment process was recorded. Cardiac function was monitored in all patients every 3 months by electrocardiogram, and LVEF was assessed by echocardiography. Cardiotoxicity was defined as a decrease in LVEF of $>10 \%$ from baseline or a decrease to an LVEF $<50 \%$ at any time.

Breast cancer first relapse events were registered as local, contralateral, or distant when they occurred, respectively, in the same breast as the first tumor, in the other breast, or in another part of the body (brain, bone,

Table 1. Baseline Characteristics and Tumour Properties of Patients

\begin{tabular}{|c|c|}
\hline Variables & $\mathrm{N}(\%)$ \\
\hline \multicolumn{2}{|l|}{ Age (years) } \\
\hline Mean age $\pm S D$ & $51.8 \pm 10.7$ \\
\hline$\leq 35$ & $13(6.2)$ \\
\hline $36-65$ & $178(84.8)$ \\
\hline$>65$ & $19(9.0)$ \\
\hline \multicolumn{2}{|l|}{ Menopause } \\
\hline Premenopausal & $59(28.1)$ \\
\hline Postmenopausal & $151(71.9)$ \\
\hline \multicolumn{2}{|l|}{ Surgery } \\
\hline Lumpectomy & $57(27.1)$ \\
\hline Mastectomy & $153(72.9)$ \\
\hline \multicolumn{2}{|l|}{ Stage } \\
\hline IA & $14(6.7)$ \\
\hline IB & $8 \quad(3.8)$ \\
\hline IIA & $75(35.7)$ \\
\hline IIB & $51(24.3)$ \\
\hline IIIA & $30(14.3)$ \\
\hline IIIB & 14 (6.7) \\
\hline IIIC & $18 \quad(8.6)$ \\
\hline \multicolumn{2}{|l|}{ Histopathology } \\
\hline Invazive ductal carcinoma & $190(90.5)$ \\
\hline Invazive lobular carcinoma & $6(2.9)$ \\
\hline Other* & $14(6.7)$ \\
\hline \multicolumn{2}{|l|}{ Tumour size (mm) } \\
\hline $\mathrm{T} 1$ & $65(31.0)$ \\
\hline $\mathrm{T} 2$ & $131(62.4)$ \\
\hline$\geq \mathrm{T} 3$ & $14(6.7)$ \\
\hline \multicolumn{2}{|l|}{ Number of lymph node metastasis } \\
\hline 0 & $79(37.6)$ \\
\hline $1-3$ & $61(29.0)$ \\
\hline $4-9$ & $36(17.1)$ \\
\hline$>9$ & $31(14.8)$ \\
\hline Unknown & $3(1.4)$ \\
\hline \multicolumn{2}{|l|}{ Estrogen receptor (ER) } \\
\hline Negative & $100(47.6)$ \\
\hline Positive & $110(52.4)$ \\
\hline \multicolumn{2}{|l|}{ Progesterone receptor (PR) } \\
\hline Negative & $122(58.1)$ \\
\hline Positive & $88(41.9)$ \\
\hline \multicolumn{2}{|l|}{ Hormone receptor status } \\
\hline ER and PR negative & $96(45.7)$ \\
\hline ER and/or PR Positive & $114(54.3)$ \\
\hline \multicolumn{2}{|l|}{ Histologic grade } \\
\hline 1 & $6 \quad(2.9)$ \\
\hline 2 & 77 (36.7) \\
\hline 3 & $109(51.9)$ \\
\hline Unknown & 18 (8.6) \\
\hline
\end{tabular}

*Mixed tumours, medullary tumors; Histologic grade; $1=$ Well differentiated; Grade 2= Moderately differentiated; Grade 3=Poorly differentiated 
liver, lung, visceral, lymph nodes, and other organs). Relapse Free Survival (RFS) was defined as time from diagnosis to local, regional, or distant relapse, or death from breast cancer. Overall survival was defined as the time relapsing from diagnosis to death or final evaluation in the clinic and calculated accordingly.

\section{Statistical analysis}

Statistical data were analysed using, SPSS 15.0 software program. Fisher and chi square tests were used for nominal variables and numerical data. Kaplan-Meier analysis was employed for survival rates and comparisons were made with log-rank test. Univariate and multivariate analyses were evaluated with Cox regression model. $\mathrm{p}<$ 0.05 value was considered statistically significant.

\section{Results}

\section{Patients characteristics}

In this retrospective study, 210 HER 2 positive early stage breast cancer patients were detected. The number of patients with IHC score 3+ was 156 (74.3\%) and FISH analysis was not carried out. The number of patients with IHC score 2+ who were found to be HER2 amplification positive with FISH or SISH analysis was 54 (25.7\%). Mean age of the patients was 51.8 and $71.9 \%$ was postmenopausal.

$72.9 \%$ of the patients underwent radical mastectomy. $37.6 \%$ of patients had negative nodes and \% 31 had T1 tumor size and $52.4 \%$ was positive for ER receptor. The rate of either ER or PR positivity was $54.3 \%$. As expected for HER 2 positive carcinomas, tumors were mainly grade III (51.9\%) and ductal carcinoma (90.5\%). Table 1 lists the characteristics of patients and tumors.

\section{Adjuvant treatments}

In all patients, adjuvant trastuzumab based treatment was planned for one year at the onset. As in the HERA trial, trastuzumab had been administered, mainly every 3 weeks,

Table 2. Adjuvant Treatments

\begin{tabular}{lr}
\hline Treatments & $\mathrm{N}(\%)$ \\
\hline Chemotherapy & \\
Antracyclines & $51(24.3)$ \\
Taxanes & $6(2.9)$ \\
Antracyclines+Taxanes & $(72.9)$ \\
Hormone therapy & \\
Tamoxifen & $47(22.4)$ \\
Anastrazole & $9(4.3)$ \\
Letrozole & $58(27.6)$ \\
None & $96(45.7)$ \\
Radiotherapy & \\
Yes & $166(79.0)$ \\
No & $44(21.0)$ \\
& \\
Radiotherapy & \\
Concurrently with trastuzumab therapy & $93(56.0)$ \\
Simultaneous with trastuzumab therapy & $73(44.0)$ \\
Duration of Trastuzumab Therapy & \\
1 years & $188(89.5)$ \\
From $\geq 9$ to < 12 months & $18(8.5)$ \\
From $\geq 3$ to < 9 months & $4(1.9)$ \\
\hline
\end{tabular}

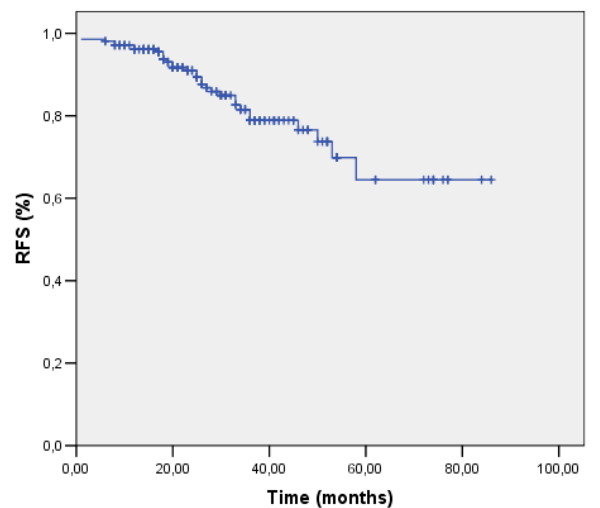

Figure 1. Relapse Free Survival for Patients with HER2- Positive Early Breast Cancer Receiving Adjuvant Trastuzumab

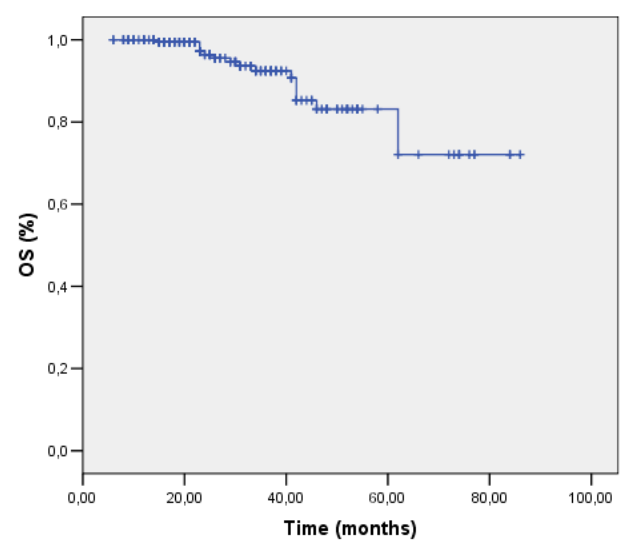

Figure 2. Overall Survival for Patients with HER2Positive Early Breast Cancer Receiving Adjuvant Trastuzumab

in patients with primary breast cancer following surgery, adjuvant chemotherapy, and radiotherapy (if applicable). Trastuzumab treatment was commenced with an $8 \mathrm{mg}$ / $\mathrm{kg}$ loading dose followed by a $6 \mathrm{mg} / \mathrm{kg}$ maintenance dose every three weeks for one year duration. $72.9 \%$ of the patients received taxane and anthracycline based chemotherapy while $24.3 \%$ received only anthracycline based chemotherapy and $2.9 \%$ only adjuvant taxane and trastuzumab treatment. $79.0 \%$ underwent radiotherapy treatment following mastectomy. $44 \%$ of the patients received radiotherapy along with trastuzumab. Finally, $44.3 \%$ received hormone treatment ( Table 2).

Cardiotoxicity developed in $7(3.3 \%)$ patients during trastuzumab treatment. Of these patients, 6 were asymptomatic and cardiotoxicity was detected with echocardiography. It was established that cardiotoxicity developed after median 9 cycles (range, 3-15 cycles). When serious symptomatic heart failure was detected in one patient, her treatment was completely discontinued. All patients in whom cardiotoxicity developed were those who previously received antracycline based chemotherapy.

\section{Survival}

The median follow up was 27.5 months $(6.0-86.0$ months). RFS was 68.0 months (95\% CI: 62.1-74.0) and OS was found to be 74.8 months (95\% CI: 69.5-80.1). The 3 year OS for all patients $92.0 \%$ and RFS was $79.6 \%$ (Figures 1 and 2). 
Table 3. Univariate and Multivariate Analyses of Parameters for Relaps Free Survival

\begin{tabular}{|c|c|c|c|c|c|c|}
\hline \multirow[t]{2}{*}{ Parameters } & \multicolumn{3}{|c|}{ Univariate analyses } & \multicolumn{3}{|c|}{ Multivariate analyses } \\
\hline & HR & $95 \% \mathrm{CI}$ & $\mathrm{P}$ & $\mathrm{HR}$ & $95 \% \mathrm{CI}$ & $\mathrm{P}$ \\
\hline Menopause (yes vs. no) & 0.6 & $0.3-1.3$ & 0.24 & & & \\
\hline Surgery (mastectomy $v s$ breast-conserving surgery) & 0.6 & $0.2-1.4$ & 0.25 & & & \\
\hline Histology (duktal carcinoma vs others*) & 0.8 & $0.2-2.6$ & 0.70 & & & \\
\hline Tumour size $(>\mathrm{T} 1$ vs $\leq \mathrm{T} 1$ & 2.8 & $1.1-8.0$ & 0.05 & 1.4 & $0.5-4.2$ & 0.54 \\
\hline Lymph nodes ( positive $v s$. negative) & 2.1 & $0.9-4.9$ & 0.80 & & & \\
\hline Estrogen receptor (positive $v s$. negative ) & 0.7 & $0.3-1.3$ & 0.25 & & & \\
\hline Progesteron receptor (positive $v s$. negative ) & 0.2 & $0.3-1.4$ & 0.26 & & & \\
\hline Hormone receptor status (positive vs. negative ) & 0.6 & $0.3-1.3$ & 0.20 & & & \\
\hline Grade (III vs. II/I) & 2.1 & $1.1-4.2$ & 0.04 & 2.1 & $0.9-4.8$ & 0.07 \\
\hline Anthracycline-taxanes $v s$. anthracycline-no taxanes & 0.9 & $0.4-1.9$ & 0.86 & & & \\
\hline Hormone therapy (yes vs no) & 0.6 & $0.3-1.6$ & 0.11 & & & \\
\hline Radiotherapy ( yes $v s$ no) & 0.7 & $0.3-1.9$ & 0.54 & & & \\
\hline Concurrently $v s$. simultaneously with trastuzumab therapy & 0.7 & $0.3-1.8$ & 0.44 & & & \\
\hline
\end{tabular}

In hormone receptor (HR) positive patients (ER and / or PR positive), rate of 3 years OS was $93 \%$ and 3 years RFS was $87 \%$. Among HR negative patients (ER neg and PR neg) 3 years OS was $90 \%$ and 3 years RFS was $71 \%$. There was no significant difference between HR positive and HR negative patients $(\mathrm{p}<0.05)$. In lymph node positive patients, 3 years OS was $88 \%$ and 3 years RFS was $73 \%$ while in lymph node negative patients, the corresponding rates were respectively, $96 \%$ and $89 \%$.

Of overall 210 patients, $188(89.5 \%)$ completed planned adjuvant one year trastuzumab treatment. Treatment was interrupted in 21 patients due to relapse during treatment process and in one patient to cardiotoxicity. During median 27.2 months follow up period, metastasis did not develop in $180(85.7 \%)$ patients while local recurrence occurred in $8(3.8 \%)$ patients and metastasis developed in $30(14.3 \%)$ patients. In $14(6.7 \%)$ patients, metastasis developed under trastuzumab treatment. In these patients, the initial site of metastasis was as follows: brain in $7(3.3 \%)$ patients, bone in $7(3.3 \%)$ patients, liver in $5(2.4 \%)$ patients, lung in 5 $(2.4 \%)$ patients, and skin in $3(1.4 \%)$ patients. In addition, there was also thyroid metastasis concurrent with brain metastasis in one patient. In all patients, time to relapse was median 21.5 months (range; 1.4 to 53.0 months) and in those relapsing during trastuzumab treatment 6.2 months (range, 1.4 to 9.9 months).

In univariate analysis performed for RFS, no statistically significant relation with age, menopausal status, surgical method, histopathology, nodal involvement, estrogen receptor status, progesterone receptor status, chemotherapy regime with taxane or antracycline or with taxane and antracycline, hormonal treatment status, radiotherapy treatment. Univariate analyses indicated larger tumour size and grade III were significantly associated $(\mathrm{p}<0.05)$ with RFS. Multivariate analyses of covariates displaying $\mathrm{p}<0.05$ identified grade III $(\mathrm{HR}=2.1$, $95 \%$ CI $0.9-4.8, \mathrm{p}=0.07)$ as independent prognostic factor (Table 3).

\section{Discussion}

The treatment of breast cancer has advanced significantly since 1990 , the most important advance being trastuzumab treatment heralding a new era for HER 2 positive breast cancer patients. In early stage HER2 positive breast cancer patients, the advent of adjuvant trastuzumab treatment improved outcomes considerably. In the present study, it was found that trastuzumab treatment was feasible and well tolerated in routine clinical practice.

In the present study, median duration of follow up was 27.5 months and, 3 year RFS and OS values were $79.6 \%$ and $92.0 \%$, respectively. These survival results are consistent with those of HERA; which is the largest adjuvant trastuzumab study performed in Europe (Smith et al., 2007). All of our patients received trastuzumab treatment every three weeks following locoregional treatment and chemotherapy and $89.5 \%$ completed planned one year treatment. Only in one patient, was the treatment interrupted owing to drug associated toxicity. Compliance with treatment and safety was consistent with that reported in the literature (Smith et al., 2007; Palmieri et al., 2011; Campiglio et al., 2013; Tai et al., 2013).

Even though there is significant improvement in survival in HER2 positive patients with adjuvant trastuzumab treatment, relapse may occur quite frequently (Mariani et al., 2009). In the present study, the rate of relapse was $14.3 \%$, which is higher than that reported in the previous studies. For example, in 2 year results of HERA, this figure was reported to be $12 \%$ (5). In another adjuvant trastuzumab study carried out with 284 patients, the rate of relapse was reported to be $10.3 \%$ and median time to relapse 8.7 months (Krell et al., 2011). In the present study, relapses were established to occur within the first six months.

The most common sites of metastasis were brain and bone in the present study. Sites of distant metastasis, recurrence pattern and CNS involvement were consistent with the findings of previous adjuvant studies (PiccartGebhart et al., 2005; Metzger-Filho et al., 2013). In HER2 positive breast cancer patients, CNS disease is an important source of concern. In a metanalysis performed, significant increase was shown in the risk of brain metastasis (Bria et al., 2008). In patients who received adjuvant trastuzumab, the first event was CNS metastasis while in those who do not receive this treatment, metastasis occurred in other 
sites earlier (Romond et al., 2005). Probable explanation may be that the ability of trastuzumab to control systemic disease is stronger in areas other than CNS. The frequency of CNS metastatic disease may be linked to inadequate passage of trastuzumab from blood brain barrier as reported in previous studies (Krell et al., 2011).

In the present study, bone metastasis was more common than that in other studies. In order to assess whether the effect of trastuzumab on bone metastasis is low, penetration of the drug to this tissue may be analysed. Owing to the heterogenity of the tumor, confirming HER2 expression levels in the tumor cells in the bone may be useful. As reported in four year follow up results of the HERA study, in the present study trastuzumab exerted the most beneficial effect in liver recurrences (Metzger-Filho et al., 2013).

In HER 2 positive early stage disease, data on subgroups with high risk of recurrence and death in spite of optimal treatment is not adequate (Dent et al., 2007; Mansell et al., 2009). In the present study, in the analysis of factors influencing the response to treatment and the risk of relapse, unlike other studies, no statistically significant relation was found between RFS and clinical and pathological paramaters except for grade III tumor. These results are different from those of previous analyses on factors predicting relapse (Smith et al., 2007; Gianni et al., 2011). In previous studies on adjuvant trastuzumab, lymph node positivity and ER positivity were found to be predictive of DFS (Campiglio et al., 2013; Perez et al., 2011). In the updated analysis of NSABP B-31 and NCCTG N9831 studies for DFS, it was reported that hormone receptor positivity, tumor size over $2 \mathrm{~cm}$ and lymph node positivity were predictive of DFS. The combined data also showed the prognostic impact of hormone receptor status on DFS and OS in a multivariate analysis (Perez et al., 2014). Hormone receptor status is the most important discriminator of breast cancer subtypes (Prat and Perou, 2010). Although it is known that trastuzumab treatment is effective on both HR-positive and HR- negative patients, data suggests that HER2 positive disease was influenced from HR status (Vas-Luis et al., 2013). In the analysis of National Comprehensive Cancer Network Breast Cancer Outcomes database, HER2 positive/HR negative disease had a higher risk of death within 5 years of initial diagnosis compared with patients with HR-positive / HER2-positive disease (Vaz-Luis et al., 2012). In another study, it was demonstrated that clinical benefit lasted longer in HR positive tumors (Vaz-Luis et al., 2013). In the present study, although HR positive patients had longer median RFS and OS compared to HR negative patients, the difference was not statistically significant.

Tumor size (T), defined as the largest diameter of the primary breast tumor, was early recognized as an important prognostic factor in breast cancer (Koscielny et al., 1984; Carter, Allen and Henson, 1989). Yet, in the present study, while tumor size was found to be influential on RFS in univariate analysis, its influence was not seen anymore in multivariate analysis.

Compatible with the present study, in NSABP B-31 and NCCTG N9831 studies $70 \%$ of the patients were in grade 3, evaluated as a unfavorable prognostic factor (Perez et al., 2014) Histological grade, shows a very strong correlation with prognosis; patients with grade I tumours have a significantly better survival than those with grade II and III tumours ( $<<0.0001)$ (Elston and Ellis, 1991).

In the present study, the occurrence of relapse during adjuvant trastuzumab treatment may be ascribed to many factors. The development of relapse during adjuvant trastuzumab treatment indicates de novo trastuzumab resistance, hence trastuzumab treatment may not yield more benefit (Pohlmann, Mayer and Mernaugh, 2009). As reported previously, stronger effect of trastuzumab along with chemotherapy, the change in receptor status with exposure to trastuzumab, HER2 negative phenotype becoming more potential are among the probable causes cited (Mittendorf et al., 2009). In a study reporting relapse risk at the rate of $11 \%$ (Campiglio et al., 2013), it is proposed that as the distribution of pathological parameters is the same in early and late relapses, these parameters are not associated with trastuzumab resistance (Gianni et al., 2011; Campiglio et al., 2013). We also believe that lower number of patients in the present study and short duration of follow up may have influenced the results.

In the present study, the rate of cardiotoxicity associated with trastuzumab treatment was found to be $3.3 \%$, which was similar to that reported in the previous metanalyses (Smith et al., 2007; Suter et al., 2007; Bria et al., 2008; Dahabreh et al., 2008). All of our patients with the development of cardiotoxicity had previously received antracycline based chemotherapy. The event was reversible in all but one of patients with the development of cardiotoxicity. Compliance with treatment and tolerability was quite satisfactory. In a recent population based observational study, it was reported that the risk in cardiotoxicity was obvious in trastuzumab treatment following antracycline and it became more marked over time (Bowles et al., 2012). In patients receiving RT concurrent with trastuzumab, no short term cardiotoxicity was observed, as has been reported by others (Shaffer et al., 2009). Since the majority of patients receive trastuzumab following antracycline based regimen, cardiac monitorization is imperative during treatment in clinical practice. Long term monitorization is necessary in order to evaluate probable long term toxicity.

Retrospective design of the present study, relatively small sample size and short duration of follow up are among its limitations. In addition, it was not possible to reevaluate receptor status in tumor samples. We reported our findings based upon the fact that majority of early adjuvant studies reported two year outcomes. We believe that evaluation of response to treatment and cardiotoxicity in the present study is more reliable, as it included data only in two oncology centers, unlike the previous multicenter studies, and treatment and follow up was carried out at the same center.

In conclusion; as reported in the previous studies of adjuvant trastuzumab, it was demonstrated in the present study that survival results were improved especially in HER2-positive early stage breast cancer, which is especially agressive and known to recur within a few 
years of the diagnosis. Satisfactory safety profile and treatment efficacy in the present studyt were in keeping with that reported in previous clinical studies. Of all classical prognostic factors, only tumor grade 3 was found to have prognostic significance. The occurrence of relapse with adjuvant trastuzumab clearly indicates the need for determining molecular predictors, which will define this group better, help to avoid unnecessary treatment and explain the resistance to HER2 based treatment better. In the future, larger prospective studies, which will evaluate the molecular characteristics of subtypes with relapse, are required.

\section{Acknowledgements}

The authors have declared no conflicts of interest.

\section{References}

Abe H, Mori T, Kawai Y, et al (2013). Safety assessment of intravenous administration of trastuzumab in $100 \mathrm{ml}$ saline for the treatment of HER2- positive breast cancer patients. Asian Pac J Cancer Prev, 14, 4843-6.

Baselga J, Perez EA, Pienkowski T, Bell R (2006). Adjuvant trastuzumab: a milestone in the treatment of HER-2-positive early breast cancer. Oncologist, 11, 4-12

Bria E, Cuppone F, Fornier M, et al (2008). Cardiotoxicity and incidence of brain metastases after adjuvant trastuzumab for early breast cancer: the dark side of the moon? A metaanalysis of the randomized trials. Breast Cancer Res Treat, 109, 231-9.

Bowles EJ, Wellman R, Feigelson HS et al (2012). Risk of heart failure in breast cancer patients after anthracycline and trastuzumab treatment: a retrospective cohort study. $J$ Natl Cancer Inst, 104, 1293-1305.

Campiglio M, Bufalino R, Sasso M, et al (2013). Effect of adjuvant trastuzumab treatment in conventional clinical setting: an observational retrospective multicenter Italian study. Breast Cancer Res Treat, 141, 101-10.

Carter CL, Allen C, Henson DE (1989). Relation of tumor size, lymph node status, and survival in 24,740 breast cancer cases. Cancer, 63, 181.

Dahabreh IJ, Linardou H, Siannis F, et al (2008). Trastuzumab in the adjuvant treatment of early-stage breast cancer: a systematic review and meta-analysis of randomized controlled trials. Oncologist, 13, 620-630.

Dent R, Trudeau M, Pritchard KI, et al (2007). Triple-negative breast cancer: clinical features and patterns of recurrence. Clin Cancer Res, 13, 4429 -34.

Elston CW, Ellis IO (1991). Pathological prognostic factors in breast cancer. I. The value of histological grade in breast cancer: experience from a large study with long-term followup. Histopathology, 19, 403.

Gabos Z, Thoms J, Ghosh S, et al (2010). The association between biological subtype and locoregional recurrence in newly diagnosed breast cancer. Breast Cancer Res Treat, 124, 187-94.

Gianni L, Dafni U, Gelber RD et al (2011). Treatment with trastuzumab for 1 year after adjuvant chemotherapy in patients with HER2-positive early breast cancer: a 4-year follow-up of a randomised controlled trial. Lancet Oncol, 12, 236-244.

Goldhirsch A, Piccart-Gebhart MJ, Procter et al (2013). HERA TRIAL: 2 years versus 1 year of trastuzumab after adjuvant chemotherapy in women with HER2-positive early breast cancer at 8 years of median follow up. Cancer Res, 72, 103

Koscielny S, Tubiana M, Le MG, et al (1984). Breast cancer: relationship between the size of the primary tumour and the probability of metastatic dissemination. Br J Cancer, 49, 709 .

Krell J, James CR, Shah D, et al (2011). Human epidermal growth factor receptor 2-positive breast cancer relapsing post-adjuvant trastuzumab: pattern of recurrence, treatment and outcome. Clin Breast Cancer, 11, 153-60.

Mansell J, Monypenny IJ, Skene AI, et al (2009). Patterns and predictors of early recurrence in postmenopausal women with estrogen receptor-positive early breast cancer. Breast Cancer Res Treat, 117, 91-8

Mariani G, Fasolo A, De Benedictis E, et al (2009). Trastuzumab as adjuvant systemic therapy for HER2-positive breast cancer. Nat Clin Pract Oncol, 6, 93-104.

Metzger-Filho O, Procter M, De Azambuja AE et al (2013). Magnitude of trastuzumab benefit in patients with HER2positive, invasive lobular breast carcinoma: results from the HERA trial. J Clin Oncol, 31, 1954-60.

Mittendorf EA, Wu Y, Scaltriti M, et al (2009). Loss of HER2 amplification following trastuzumab-based neoadjuvant systemic therapy and survival outcomes. Clin Cancer Res, 15, 7381-8.

Palmieri C, Shah D, Krell J et al (2011). Management and outcome of HER2-positive early breast cancer treated with or without trastuzumab in the adjuvant trastuzumab era. Clin Breast Cancer, 11, 93-102.

Perez EA, Romond EH, Suman VJ, et al (2011). Four year follow-up of trastuzumab plus adjuvant chemotherapy for operable human epidermal growth factor receptor 2-positive breast cancer: joint analysis of data from NCCTG N9831 and NSABP B-31. J Clin Oncol, 29, 3366-73.

Perez EA, Romond EH, Suman VJ, et al (2014). Trastuzumab Plus adjuvant chemotherapy for human epidermal growth factor receptor 2-positive breast cancer: planned joint analysis of overall survival from NSABP B-31 and NCCTG N9831. J Clin Oncol, 32, 3744-52.

Perou CM, Sorlie T, Eisen MB, et al (2000). Molecular portraits of human breast tumours. Nature, 406, 747-52.

Piccart-Gebhart M, Procter M, Leyland-Jones B, et al (2005). Trastuzumab after adjuvant chemotherapy in HER2-positive breast cancer. $N$ Engl J Med, 353, 1659-72.

Pohlmann PR, Mayer IA, Mernaugh R (2009). Resistance to trastuzumab in breast cancer. Clin Cancer Res, 15, 7479-91

Prat A, Perou CM (2010). Deconstructing the molecular portraits of breast cancer. Mol Oncol, 5, 5-23.

Romond EH, Perez EA, Bryant J, et al (2005). Trastuzumab plus adjuvant chemotherapy for operable HER2-positive breast cancer. $N$ Engl J Med, 353, 1673-84.

Shaffer R, Tyldesley S, Rolles M, et al (2009). Acute cardiotoxicity with concurrent trastuzumab and radiotherapy including internal mammary chain nodes: a retrospective single-institution study. Radiother Oncol, 90, 122-6.

Slamon DJ, Leyland-Jones B, Shak S, et al (2001). Use of chemotherapy plus a monoclonal antibody against her 2 for metastatic breast cancer that overexpresses her 2. $N$ Engl $J$ Med, 344, 783-892.

Smith I, Procter M, Gelber RD et al (2007). 2-Year follow up of trastuzumab after adjuvant chemotherapy in HER2- positive breast cancer: a randomised controlled trial. Lancet, 369, 29-36

Spielmann M, Roché H, Delozier T, et al (2009). Trastuzumab for patients with axillary node- positive breast cancer: results of the FNCLCC-PACS 04 trial. J Clin Oncol, 27, 6129-34.

Suter TM, Procter M, van Veldhuisen DJ, et al (2007). Trastuzumab-associated cardiac adverse effects in the 

herceptin adjuvant trial. J Clin Oncol, 25, 3859-65.

Tai CJ, Pan CK, Chen CS et al (2013). Adjuvant trastuzumab for 6 months is effective in patients with HER2-positive stage II or III breast cancer. Asian Pac J Cancer Prev, 14, 1981-4.

Vaz-Luis I, Winer EP, Lin NU et al (2013). Human epidermal growth factor receptor-2-positive breast cancer: does estrogen receptor status define two distinct subtypes? Ann Oncol, 24, 283-91

Vaz-Luis I, Ottesen RA, Hughes ME, et al (2012). Impact of hormone receptor status on patterns of recurrence and clinical outcomes among patients with human epidermal growth factor-2-positive breast cancer in the National Comprehensive Cancer Network: a prospective cohort study. Breast Cancer Res, 1, 129.

Vaz-Luis I, Seah D, Olson EM, et al (2013). Clinicopathological features among patients with advanced human epidermal growth factor-2-positive breast cancer with prolonged clinical benefit to first-line trastuzumab-based therapy: a retrospective cohort study. Clin Breast Cancer, 13, 254-63.

Zhu ZL, Zhang J, Chen ML, Li K (2013). Efficacy and safety of Trastuzumab added to standard treatments for HER2positive metastatic breast cancer patients. Asian Pac J Cancer Prev, 14, 7111-6. 